\title{
Probability, Parallelism and the State Space Exploration Problem
}

\author{
William Knottenbelt ${ }^{1}$, Mark Mestern ${ }^{2}$, Peter Harrison ${ }^{1}$, and Pieter Kritzinger ${ }^{2}$ \\ 1 Department of Computing, Imperial College, 180 Queens Gate, London SW7 2BZ, \\ United Kingdom, email: \{wjk,pgh\}@doc.ic.ac.uk \\ 2 Computer Science Department, University of Cape Town, Rondebosch 7701, \\ South Africa, email: \{mmestern,psk\}@cs.uct.ac.za
}

\begin{abstract}
We present a new dynamic probabilistic state exploration algorithm based on hash compaction. Our method has a low state omission probability and low memory usage that is independent of the length of the state vector. In addition, the algorithm can be easily parallelised. This combination of probability and parallelism enables us to rapidly explore state spaces that are an order of magnitude larger than those obtainable using conventional exhaustive techniques. We implement our technique on a distributed-memory parallel computer and we present results showing good speedups and scalability. Finally, we discuss suitable choices for the three hash functions upon which our algorithm is based.
\end{abstract}

\section{Introduction}

Complex systems can be modelled using high-level formalisms such as stochastic Petri nets and process algebras. Often the first phase in the logical and numerical analysis of these systems is the explicit generation and storage of the model's underlying state space and state transition graph. In special cases, where the state space has sufficient structure, an efficient analytical solution can be obtained without the explicit enumeration of the entire state space. Several ingenious techniques, predominantly based on the theory of queueing networks, can be applied in such cases [3]. Further, certain restricted hierarchical structures allow states to be aggregated and the state space to be decomposed $[5,16]$. In this paper, however, we consider the general problem where no symmetry or other structure is assumed.

Conventional state space exploration techniques have high memory requirements and are very computationally intensive; they are thus unsuitable for generating the very large state spaces of real-world systems. Various authors have proposed ways of solving this problem by either using shared-memory multiprocessors [2] or by distributing the memory requirements over several computers in a network $[7,6]$.

Allmaier et al. [2] present a parallel shared memory algorithm for the analysis of Generalised Stochastic Petri Nets (GSPNs) [1]. The shared memory approach means that there is no need to partition the state space as must be done in the case of distributed memory. This also brings the advantage of simplifying the 
load balancing problem. However, it does introduce synchronisation problems between the processors. Their technique is tested on a Convex SPP 1600 shared memory multiprocessor with $4 \mathrm{~GB}$ of main memory. The authors observe good speedups for a range of numbers of processors employed and the system can handle 4000000 states with 2 GB of memory.

Caselli et al. [6] offer two ways to parallelise the state space generation for massively parallel machines. In the data-parallel method, a marking of a GSPN with $t$ transitions is assigned to $t$ processors. Each processor handles the firing one transition only and is responsible for determining the resulting state. This method was tested on a Connection Machine CM-5 and showed computation times linear in relation to the number of states. In the message-passing method the state space is partitioned between processors by a hash function and newly discovered states are passed to their respective processors. This method achieved good speedups on the CM-5, but was found to be subject to load imbalance.

Ciardo et al. [7] present an algorithm for state space exploration on a network of workstations. Their approach is not limited to GSPNs but has a general interface for describing state transition systems. Their method partitions the state space in a way similar to [6] but no details on the storage techniques used are given. The importance of a hashing function which evenly distributes the states across the processors is emphasised, but the method also attempts to reduce the number of states sent between processors. It was tested on a network of SPARC workstations interconnected by an Ethernet network and an IBM SP2 multiprocessor. In both cases a good reduction in processing time was reported although with larger numbers of processors, diminishing returns occurred. The largest state space successfully explored had 4500000 states; this required four hours of processing on a 32-node IBM SP-2.

All the techniques proposed so far do not take advantage of the considerable gains achieved by using dynamic storage techniques based on hash compaction. The dynamic storage method we present here has several important advantages: memory consumption is low, space is not wasted by a static allocation and access to states is simple and rapid. We also present a parallel version of our technique which results in further performance gains.

After introducing the problem of state space exploration in Section 2, we give the details of the storage allocation algorithm in Section 3 and of the parallel state space generation algorithm in Section 4. Numerical results on the performance of the algorithm are in Section 5 and Section 6 discusses suitable hashing and partition functions. Section 7 concludes and considers future work.

\section{State Space Exploration}

Fig. 1 shows an outline of a simple sequential state space exploration algorithm. The core of the algorithm performs a breadth-first search (BFS) traversal of a model's underlying state graph, starting from some initial state $s_{0}$. This requires two data structures: a FIFO queue $F$ which is used to store unexplored states and a table of explored states $E$ used to prevent redundant state exploration. 


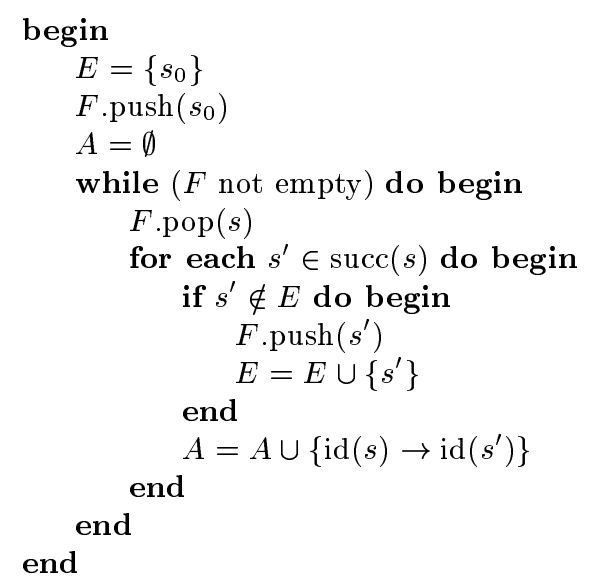

Fig. 1. Sequential state space generation algorithm

The function $\operatorname{succ}(s)$ returns the set of successor states of $s$. Some formalisms (such as GSPNs) include support for "instantaneous events" which occur in zero time. A state which enables an "instananeous event" is known as a vanishing state. We will assume that our successor function implements one of several known on-the-fly techniques available for eliminating vanishing states [8] [17]. In addition, we will not consider the case where $s_{0}$ is vanishing.

As the algorithm proceeds, it constructs $A$, the state graph. To save space, the states are identified by a unique state sequence number given by the function $\operatorname{id}(s)$. If we require the equilibrium state space probability distribution, we must construct a Markov chain by storing in $A$ the transition rate between state $s$ and $s^{\prime}$ for every arc $s \rightarrow s^{\prime}$. The graph $A$ is written out to disk as the algorithm proceeds, so there is no need to store it in main memory.

\section{Dynamic Probabilistic Hash Table Compaction}

The memory consumed by the state exploration process depends on the layout and management of the two main data structures of Fig. 1. The FIFO queue can grow to a considerable size in complex models. However, since it is accessed sequentially at either end, it is possible to manage the queue efficiently by storing the head and tail sections in main memory, with the central body of the queue stored on disk. The table of explored states, on the other hand, enjoys no such locality of access, and it has to be able to rapidly store and retrieve information about every reachable state. A good design for this structure is therefore crucial to the space and time efficiency of a state generator.

One way to manage the explored state table is to store the full state descriptor of every state in the state table. Such exhaustive techniques guarantee complete 
state coverage by uniquely identifying each state. However, the high memory requirements of this approach severely limit the number of states that can be stored. Probabilistic techniques, on the other hand, use hashing techniques to drastically reduce the memory required to store states. This reduction comes at a cost, however, and it is possible that the hash table will represent two distinct states in the same way. If this should happen, the state hash table will incorrectly report a state as previously explored. This will result in incorrect transitions in the state graph and the omission of some states from the hash table. This risk may be acceptable if the probability of inadvertently omitting even one state can be quantified and kept very small.

Probabilistic methods first gained widespread popularity with the development of Holzmann's bit-state hashing technique $[13,14]$. This technique aims at maximizing state coverage in the face of limited memory by using a hash function to map each state onto a single bit position in a large bit vector. Holzmann's method was subsequently improved upon by Wolper and Leroy's hash compaction technique [19], and Stern and Dill's enhanced hash compaction method [18]. These techniques hash states onto compressed values which are inserted into a large pre-allocated hash table with a fixed number of slots.

All of these probabilistic methods rely on static memory allocation, since they pre-allocate large blocks of memory for the explored-state table. Since the number of states in the system is in general not known beforehand, the preallocated memory may not be sufficient, or may be a gross overestimation. We now introduce a new probabilistic technique which uses dynamic storage allocation and which yields a good collision avoidance probability.

The system is illustrated in Fig. 2. The explored state table takes the form of a hash table with several rows. Attached to each row is a linked list which stores compressed state descriptors. Two independent hash functions are used. The primary hash function $h_{1}(s)$ is used to determine which hash table row should be used to store a compressed state and the secondary hash function $h_{2}(s)$ is used to compute the compressed state descriptor values (also known as secondary keys). If a state's secondary key $h_{2}(s)$ is present in the hash table row given by its primary key $h_{1}(s)$, then the state is deemed to have been explored. Otherwise the secondary key is added to the hash table row and it's successors are pushed onto the FIFO queue. Note that two states $s_{1}$ and $s_{2}$ are classified as being equal if and only if $h_{1}\left(s_{1}\right)=h_{1}\left(s_{2}\right)$ and $h_{2}\left(s_{1}\right)=h_{2}\left(s_{2}\right)$; this may happen even when the two state descriptors are different, so collisions may occur (as in all other probabilistic methods).

\subsection{Reliability of the probabilistic dynamic state hash table}

We consider a hash table with $r$ rows and $t=2^{b}$ possible secondary key values, where $b$ is the number of bits used to store the secondary key. In such a hash table, there are $r t$ possible ways of representing a state. Assuming that $h_{1}(s)$ and $h_{2}(s)$ distribute states randomly and independently, each of these representations are equally likely. Thus, if there are $n$ distinct states to be inserted into the hash 


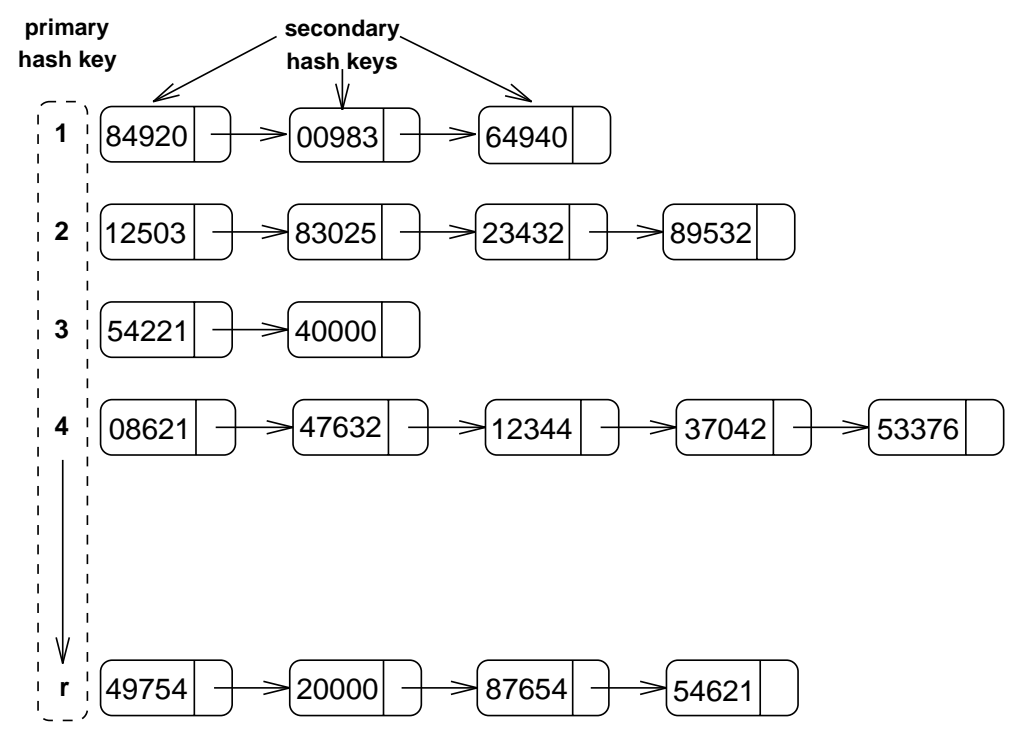

Fig. 2. Hash table with compressed state information

table, the probability $p$ that all states are uniquely represented is given by:

$$
p=\frac{(r t) !}{(r t-n) !(r t)^{n}}
$$

Using Stirling's approximation for $n$ ! in Eq. (1) yields:

$$
p \approx e^{-\frac{n^{2}}{r t}}
$$

If $n^{2}<<r t$ (as will be the case in practical schemes with $p$ close to 1 ), we can use the fact that $e^{x} \approx(1+x)$ for $|x|<<1$ to approximate $p$ by:

$$
p \approx 1-\frac{n^{2}}{r t}
$$

The probability $q$ that all states are not uniquely represented, resulting in the omission of one or more states from the state space, is of course simply:

$$
q=1-p \approx \frac{n^{2}}{r t}=\frac{n^{2}}{r 2^{b}}
$$

Thus the probability of state omission $q$ is proportional to $n^{2}$ and is inversely proportional to the hash table size $r$. Increasing the size of the compressed state descriptors $b$ by one bit halves the omission probability.

\subsection{Space complexity}

If we assume that the hash table rows are implemented as dynamic arrays, the number of bytes of memory required by the scheme is:

$$
M=h r+n b / 8 \text {. }
$$


Here $h$ is the number of bytes of overhead per hash table row. For a given number of states and a desired omission probability, there are a number of choices for $r$ and $b$ which all lead to schemes having different memory requirements. How can we choose $r$ and $b$ to minimize the amount of memory required? Rewriting Eq. (2):

$$
r \approx \frac{n^{2}}{q 2^{b}}
$$

and substituting this into Eq. (3) yields

$$
M \approx \frac{h n^{2}}{q 2^{b}}+\frac{n b}{8}
$$

Minimizing $M$ with respect to $b$ gives:

$$
\frac{\partial M}{\partial b} \approx-\frac{n^{2}(\ln 2) h}{q 2^{b}}+n / 8=0
$$

Solving for the optimal value of $b$ yields:

$$
b \approx \log _{2}\left(\frac{h n \ln 2}{q}\right)+3
$$

\begin{tabular}{|c|c|c|c|c|c|c|c|c|c|}
\hline \multirow{3}{*}{$q$} & \multicolumn{9}{|c|}{ number of states } \\
\hline & $10^{5}$ & \multicolumn{3}{|c|}{$10^{6}$} & \multicolumn{3}{|c|}{$10^{7}$} & \multicolumn{2}{|r|}{$10^{8}$} \\
\hline & $\mathrm{Mb} \quad b$ & $\overline{\mathrm{Mb}}$ & $b$ & $r$ & $\overline{\mathrm{Mb}}$ & $\bar{b}$ & & $\mathrm{Mb}$ & $\bar{b}$ \\
\hline 0.001 & $0.418632232\}$ & 4.60 & 35 & 04 & 50.21 & 39 & & $\overline{543.2}$ & 4222 \\
\hline 0.01 & 0.377429186 & 4.18 & 32 & 83 & 46.08 & & 38 & 502.1 & 3918 \\
\hline 0.1 & 0.336325298 & 3.77 & 29 & 26 & 41.86 & 32 & 331 & 460.8 & 352910383 \\
\hline
\end{tabular}

The corresponding optimal value of $r$ can then be obtained by substituting $b$ into Eq. (4).

Table 1. Optimal values for memory usage and the values for $b$ and $r$ used to obtain them for various system state sizes and omission probabilities $q$

Table 1 shows the the optimal memory requirements in megabytes $(\mathrm{Mb})$ and corresponding values of $b$ and $r$ for state space sizes ranging from $10^{5}$ to $10^{8}$. We have assumed a hash table row overhead of $h=8$ bytes per row. In practice, it is difficult to implement schemes where $b$ does not correspond to a whole number of bytes. Consequently, 4 -byte or 5 -byte compression is recommended.

\section{Parallel State Space Exploration}

We now investigate how our technique can be further enhanced to take advantage of the memory and processing power provided by a network of workstations 
or a distributed-memory parallel computer. We will assume that there are $N$ nodes available. Each node has its own processor and local memory and can communicate with other nodes via a network.

In the parallel algorithm, the state space is partitioned between the nodes so that each node is responsible for exploring a portion of the state space and for constructing a section of the state graph. A partitioning hash function $h_{0}(s) \rightarrow$ $(0, \ldots, N-1)$ is used to assign states to nodes, such that node $i$ is responsible for exploring the set of states $E_{i}$ and for constructing the portion of the state graph $A_{i}$ where:

$$
\begin{aligned}
& E_{i}=\left\{s: h_{0}(s)=i\right\} \\
& A_{i}=\left\{\left(s_{1} \rightarrow s_{2}\right): h_{0}\left(s_{1}\right)=i\right\}
\end{aligned}
$$

It is important that $h_{0}(s)$ achieves a good spread of states across nodes in order to achieve good load balance. Naturally, the values produced by $h_{0}(s)$ should also be independent of those produced by $h_{1}(s)$ and $h_{2}(s)$ to enhance the reliability of the algorithm.

The operation of node $i$ in the parallel algorithm is shown in Fig. 3. Each node $i$ has a local FIFO queue $F_{i}$ used to hold unexplored local states and a hash table used to store the set $E_{i}$ representing the states that have been explored locally. State $s$ is assigned to processor $h_{0}(s)$, which stores the state's compressed state descriptor $h_{2}(s)$ in the local hash table row given by $h_{1}(s)$.

As in the sequential case, node $i$ proceeds by popping a state off the local FIFO queue and determining the set of successor states. Successor states for which $h_{0}(s)=i$ are dealt with locally, while other successor states are sent to the relevant remote processors via calls to send-state $(k, g, s)$. Here $k$ is the remote node, $g$ is the identity of the parent state and $s$ is the state descriptor of the child state. The remote processors must receive incoming states via matching calls to receive-state $(k, g, s)$ where $k$ is the sender node. If they are not already present, the remote processor adds the incoming states to both the remote state hash table and FIFO queue.

For the purpose of constructing the state graph, states are identified by a pair of integers $(i, j)$ where $i=h_{0}(s)$ is the node number of the host processor and $j$ is the local state sequence number. As in the sequential case, the index $j$ can be stored in the state hash table of node $i$. However, a node will not be aware of the state identity numbers of non-local successor states. When a node receives a state it returns its identity to the sender by calling send-id $(k, g, h)$ where $k$ is the sender, $g$ is the identity of the parent state and $h$ is the identity of the received state. The identity is received by the original sender via a call to receive-id $(g, h)$.

In practice, it is inefficient to implement the communication as detailed in Fig. 3, since the network rapidly becomes overloaded with too many short messages. Consequently state and identity messages are buffered and sent in large blocks. In order to avoid starvation and deadlock, nodes that have very few states left in their FIFO queue or are idle broadcast a message to other nodes requesting them to flush their outgoing message buffers. 


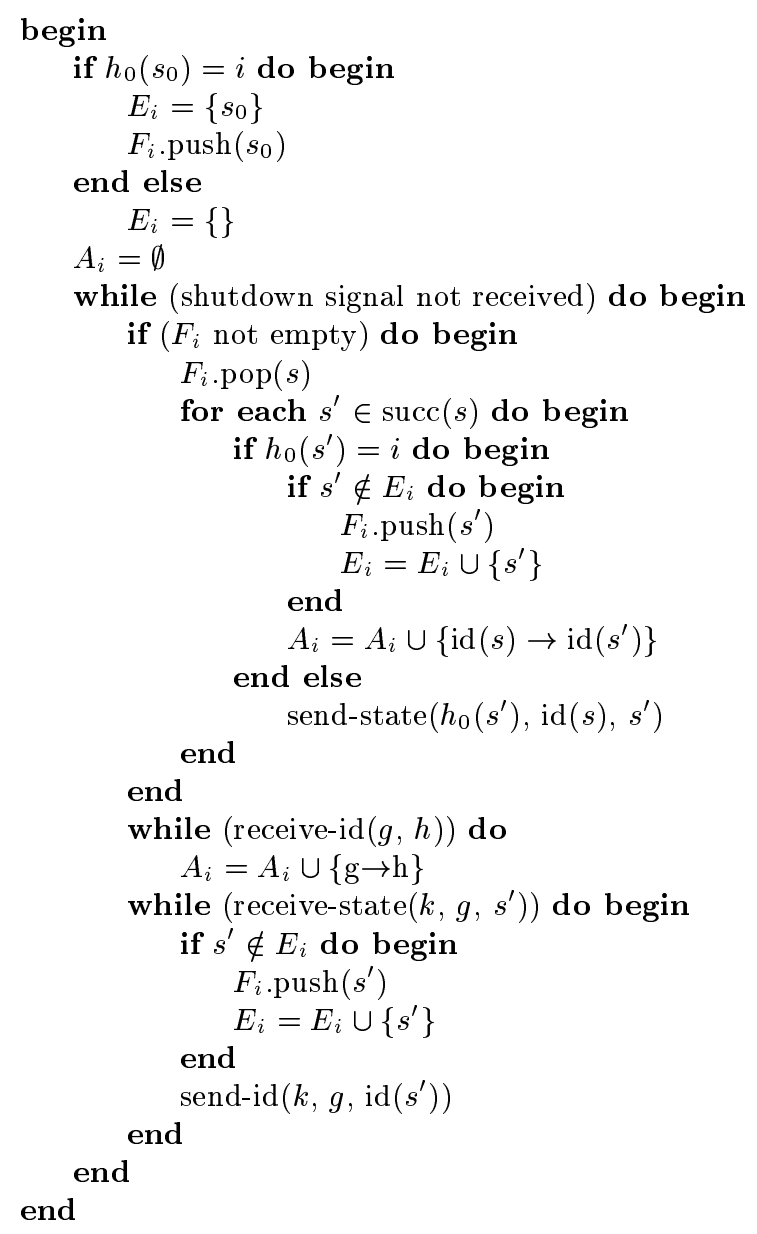

Fig. 3. Parallel state space generation algorithm for node $i$ 
The algorithm terminates when all the $F_{i}$ 's are empty and there are no outstanding state or identity messages. We use Dijkstra's circulating probe algorithm [10] to determine when this occurs.

In terms of reliability of the parallel technique, two distinct states $s_{1}$ and $s_{2}$ will mistakenly be classified as identical states if and only if $h_{0}\left(s_{1}\right)=h_{0}\left(s_{2}\right)$ and $h_{1}\left(s_{1}\right)=h_{1}\left(s_{2}\right)$ and $h_{2}\left(s_{1}\right)=h_{2}\left(s_{2}\right)$. Since $h_{0}, h_{1}$ and $h_{2}$ are independent functions, the reliability of the parallel algorithm is essentially the same as that of the sequential algorithm with a large hash table of $N r$ rows, giving a state omission probability of

$$
q=\frac{n^{2}}{N r 2^{b}}
$$

\section{Results}

To illustrate the potential of our technique, we consider a 22-place GSPN model of a flexible manufacturing system. This model, which we will refer to as the FMS model, was originally presented in detail in [9], and was subsequently used in [7] to demonstrate distributed exhaustive state space generation. A detailed understanding of the model is not required. It suffices to note that the model has a parameter $k$ (corresponding to the number of initial tokens in places $P 1, P 2$ and $P 3$ ), and that as $k$ increases, so does the number of states $n$ and the number of $\operatorname{arcs} a$ in the state graph (see Fig. 4).

\begin{tabular}{|c|c|c|}
\hline$k$ & $n$ & $\bar{a}$ \\
\hline 1 & 54 & 155 \\
\hline 2 & 810 & 3699 \\
\hline 3 & 6520 & 37394 \\
\hline 4 & 35910 & 237120 \\
\hline 5 & 152712 & 1111482 \\
\hline 6 & 537768 & 4205670 \\
\hline 7 & 1639440 & 13552968 \\
\hline 8 & 4459455 & 38533968 \\
\hline 9 & 11058190 & 99075405 \\
\hline 10 & 25397658 & 234523289 \\
\hline 11 & 54682992 & 518030370 \\
\hline 12 & 111414940 & 1078917632 \\
\hline
\end{tabular}

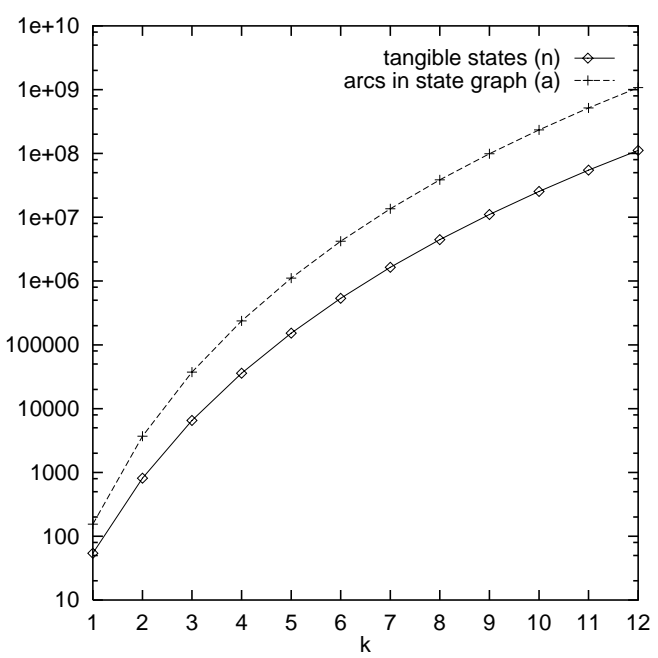

Fig. 4. The number of tangible states $(n)$ and the number of arcs in the state graph (a) for various values of $k$

We implemented the state generator algorithm of Fig. 3 using hash tables with $r=350003$ rows per processor and $b=40$ bit secondary keys. The gener- 
ator was written in $\mathrm{C}++$, with support for two popular parallel programming interfaces, viz. the Message Passing Interface (MPI) [12] and the Parallel Virtual Machine (PVM) interface [11]. Models are specified using the DNAmaca interface language [17] which allows the high-level specification of generalised timed transition systems including GSPNs, queueing networks and Queueing Petri nets [4]. The high-level specification is then translated into a $\mathrm{C}++$ class which is compiled and linked to a library implementing the core state generator. The state space and state graph are written to disk in compressed format as the algorithm proceeds.

We obtained our results on a Fujitsu AP3000 distributed-memory parallel computer with 12 processing nodes [15]. Each node has a $200 \mathrm{MHz}$ UltraSparc processor, 256Mb RAM and 4GB local disk space. The nodes run the Solaris operating system and support MPI. They are connected by a high-speed wormholerouted network with a peak throughput of $200 \mathrm{Mb} / \mathrm{s}$ (the AP-net).
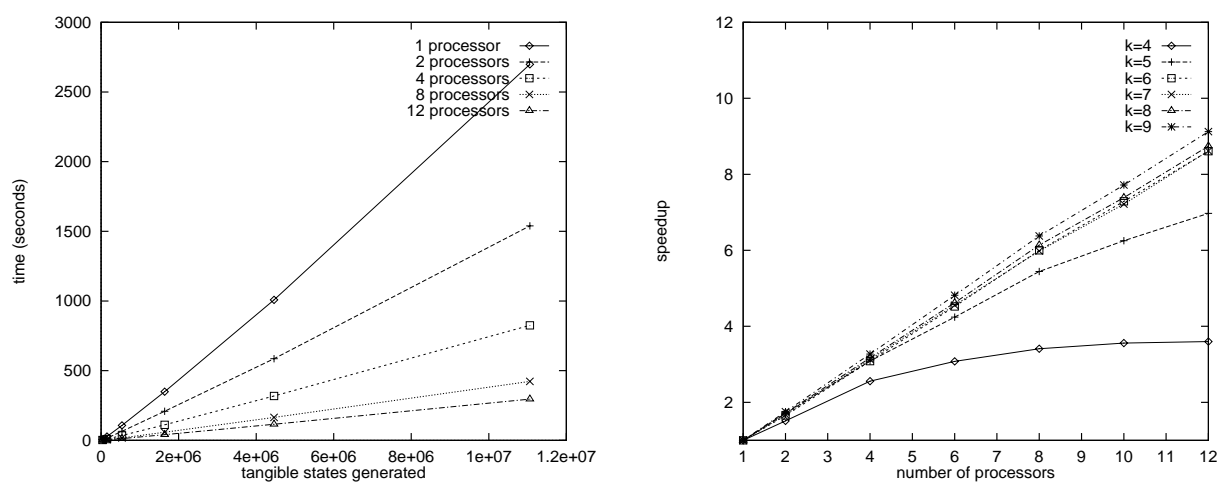

Fig. 5. Real time taken to generate state spaces up to $k=9$ using $1,2,4,8$ and 12 processors (left), and the resulting speedups for $k=4,5,6,7,8$ and 9 (right)

The graph on the left in Fig. 5 shows the time (defined as the maximum processor run time) taken to explore state spaces of different sizes (up to $k=$ 9) using 1, 2, 4, 8 and 12 processors on the AP3000. The $k=8$ state space (4459455 states) can be generated on a single processor in under 17 minutes; 12 processors require just 115 seconds. The $k=9$ state space (11058190 states) can be generated on a single processor in 45 minutes; 12 processors require just 296 seconds.

The graph on the right in Fig. 5 shows the speedups for the cases $k=$ $4,5,6,7,8,9$. The speedup for $N$ processors is given by the run time of the sequential generation $(N=1)$ divided by the run time of the distributed generation with $N$ processors. For $k=9$ using 12 processors we observe a speedup of 9.12 , giving an efficiency of $76 \%$. Most of the lost efficiency can be accounted for by communication overhead and buffer management, which is not present in 
the sequential case. Since speedup increases linearly in the number of processors for $k>6$, there is evidence to suggest that our algorithm scales well.

The memory utilization of our technique is low: a single processor generating the $k=8$ state space uses a total of $74 \mathrm{Mb}$ RAM (16.6 bytes per state), while the $k=9$ state space requires $160 \mathrm{Mb}$ RAM ( 14.5 bytes per state). 9 bytes of the memory used per state can be accounted for by the 40-bit secondary key and the 32-bit unique state identifier; the remainder can be attributed to factors such as hash table overhead and storage for the front and back of the unexplored state queue. By comparison, a minimum of 48 bytes would be required to store a state descriptor in a straightforward exhaustive implementation (22 16-bit integers plus a 32-bit unique state identifier). The difference will be even more marked with more complex models that have longer state descriptors, since the memory consumption of our technique is independent of the number of elements in the state descriptor.

Moving beyond the maximum state space size that can be generated on a single processor, the graph on the left in Fig. 6 shows the real time required to generate larger state spaces using 12 processors. For the largest case $(k=12) 55$ minutes are required to generate a state space with 111414940 tangible states and a state graph with 1078917632 arcs. The graph on the right in Fig. 6 shows the distribution of the states generated by each processor for the case $k=12$.
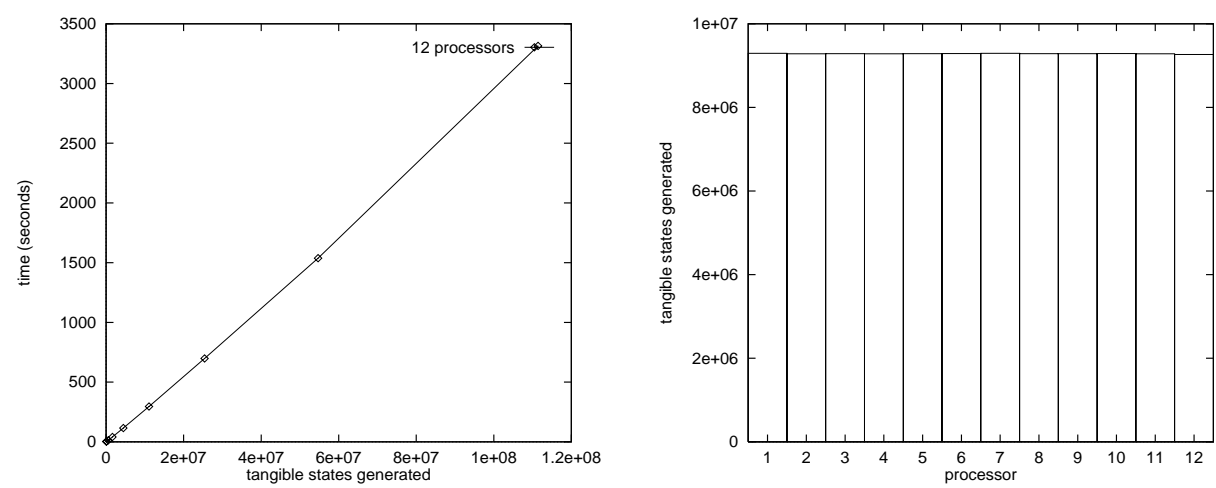

Fig. 6. Real time taken to generate state spaces up to $k=12$ using 12 processors (left) and distribution of states across processors for $k=12$ (right)

In comparison to the results reported above (see Table 4), Ciardo et al used conventional exhaustive distributed generation techniques to generate the same sample model for the case $k=8$ in 4 hours using 32 processors on an IBM SP-2 parallel computer [7]. They were unable to explore state spaces for larger values of $k$.

To enhance our confidence in our results for the case $k=12$, we use Eq. (5) to compute the probability of having omitted at least one state. For a state space 
of size $n=10^{8}$ states, the omission probability $q$ is given by:

$$
q \approx \frac{n^{2}}{N r 2^{b}}=\frac{10^{16}}{12 * 350003 * 2^{40}}=0.00217
$$

i.e. the omission probability is approximately $0.2 \%$. This is a small price to pay for the ability to explore such large state spaces, and is probably less than the chance of a serious (man-made) error in specifying the model.

To further increase our confidence in the results, we changed all three hash functions and regenerated the state space. This resulted in exactly the same number of tangible states and arcs. This process could be repeated several times to establish an even higher level of confidence in the results.

\section{Choosing good hash functions}

The reliability of our technique depends on the behaviour of the hash functions $h_{0}, h_{1}$ and $h_{2}$ in three important ways. Firstly, $h_{0}$ and $h_{1}$ should randomly partition states across the processors and hash table rows. Secondly, $h_{2}$ should result in a random distribution of compressed values. Finally, $h_{0}, h_{1}$ and $h_{2}$ should distribute states independently of one other.

Before we consider each of these functions individually, consider the two general hash functions $f_{1}$ and $f_{2}$ shown in Fig. 7 . Both map an $m$-element state vector $s=\left(s_{1}, s_{2}, \ldots, s_{m}\right)$ onto a 32-bit unsigned integer by manipulating the bit representations of individual state vector elements. The xor operator is the bitwise exclusive or operator, rol is the bitwise rotate-left operator and mod is the modulo (remainder) operator.

$f_{1}($ vector $s$, int shift $) \rightarrow$ uint 32

begin

uint32 key $=0$;

int slide $=0$;

for $i=1$ to $m$ do begin

$k e y=k e y$ xor $\left(s_{i}\right.$ rol slide $)$;

slide $=($ slide + shift $) \bmod 32 ;$

end

return key;

end $f_{2}\left(\right.$ vector $s$, int shift $_{1}$, int shift $\left._{2}\right) \rightarrow$ uint 32

begin

$$
\begin{aligned}
& \text { uint } 32 \text { key }=0 ; \\
& \text { int } \text { slide }_{1}=0, \text { slide } e_{2}=16, \text { sum }=0 \text {; } \\
& \text { for } i=1 \text { to } m \text { do begin } \\
& \quad \text { sum }=\text { sum }+s_{i} \\
& \quad \text { key }=\text { key } \text { xor }\left(s_{i} \text { rol slide } e_{1}\right) ; \\
& \quad \text { key }=\text { key xor }\left({\text { sum } \left.\text { rol } \text { slide }_{2}\right) ;} \text { slide } e_{1}=\left(\text { slide }_{1}+\text { shift }_{1}\right) \bmod 32\right. \\
& \quad \text { slide }_{2}=\left(\text { slide }_{2}+\text { shift }_{2}\right) \bmod 32 ; \\
& \text { end } \\
& \text { return key; }
\end{aligned}
$$$$
\text { end }
$$

Fig. 7. Two general hash functions for mapping states onto 32 bit unsigned integers.

Hash function $f_{1}(s$, shift $)$ uses exclusive or to combine rotated bit representations of the state vector elements. State vector element $s_{i}$ is rotated left by 
an offset of $(i \times$ shift $)$ mod 32 bits. Hash function $f_{2}\left(s\right.$, shift, shift $\left.t_{2}\right)$ is based on encoding not only element $s_{i}$ rotated left by an offset of $i \times$ shift $_{1} \bmod 32$, but also the sum $\sum_{j<i} s_{i}$ rotated left by an offset of $i \times$ shift 2 mod 32 . This technique makes the hash function resistant to any symmetries and invariants that may be present in the model.

We make use of functions $f_{1}$ and $f_{2}$ to derive suitable choices for $h_{0}(s), h_{1}(s)$ and $h_{2}(s)$ as follows:

- For the partitioning hash function, we use either

$$
h_{0}(s)=f_{1}(s, \text { shift }) \bmod \text { prime } \bmod N
$$

or

$$
h_{0}(s)=f_{2}\left(s, \text { shift } 1, \text { shift } t_{2}\right) \bmod \text { prime } \bmod N
$$

where shift, shift $t_{1}$ and shift 2 are arbitrary shifting factors relatively prime to 32 and prime is some prime number $>>N$.

- For the primary hash function, we use either

$$
h_{1}(s)=f_{1}(s, \text { shift }) \bmod r
$$

or

$$
h_{1}(s)=f_{2}(s, \text { shift } 1 \text {, shift }) \bmod r
$$

where shift, shift and $_{1}$ shift are $_{2}$ arbitrary shifting factors relatively prime to 32 and $r$, the number of rows in the hash table, is a prime number.

- For the secondary hash function, we consider 32-bit (4-byte compression) based on either $f_{1}$ or $f_{2}$ :

$$
h_{2}(s)=f_{1}(s, \text { shift })
$$

or

$$
h_{2}(s)=f_{2}\left(s, \text { shift } 1, \text { shift } t_{2}\right)
$$

where shift, shift $t_{1}$ and shift $_{2}$ are relatively prime to 32 . Function $f_{2}$ has the desirable property that it is resistant to symmetries and invariants in the model; this prevents similar (but distinct) states from having the same secondary hash values. Consequently, $f_{2}$ gives a better spread of secondary values then $f_{1}$. For 40-bit secondary hash keys (i.e. five-byte state compression), $f_{2}$ can easily be modified to produce a 40-bit hash key instead of a 32-bit hash key.

It is important to ensure the independence of the values produced by $h_{0}(s)$, $h_{1}(s)$ and $h_{2}(s)$. The following guidelines assist this:

- Some hash functions should be based on $f_{1}$ while others are based on $f_{2}$; hash functions which use the same base function should use different shifting factors.

- The hash functions should consider state vector elements in a different order.

- the value of $r$ used by $h_{1}(s)$ should not be the same as the value of prime used by $h_{0}(s)$.

The results presented in Section 5 made use of partitioning and primary functions based on $f_{1}$ and a 40-bit secondary hash function based on $f_{2}$. 


\section{Conclusion and future work}

We have presented a new dynamic probabilistic state exploration technique and developed an efficient, scalable parallel implementation. In contrast to conventional state exploration algorithms, the memory usage of our technique is very low and is independent of the length of the state vector. Since the method is probabilistic, there is a chance of state omission, but the reliability of our technique is excellent and the probability of omitting even one state is extremely small. Moreover, by performing multiple runs with independent sets of hash functions, we can reduce the omission probability almost arbitrarily at linear computational cost.

Our results to date show good speedups and scalability. It is the combination of probability and parallelism that dramatically reduces both the space and time requirements of large-scale state space exploration. We note here that the same algorithm could also be effectively implemented on a shared-memory multiprocessor architecture, using a single shared hash table and a shared breadth first search queue. There would be no need for a partitioning function and contention for rows in the shared hash table would be very small. Consequently, it should again be possible to achieve good speedups and scalability.

Our technique is based on the use of hashing functions to assign states to processors, hash table rows, and compressed state values. The reliability analysis requires that the hash functions distribute states randomly and independently and we have shown how to generate hashing functions which meet these requirements. To illustrate its potential, we have explored a state space with more than $10^{8}$ tangible states and $10^{9}$ arcs in under an hour using 12 processors on an AP3000 parallel computer. The probability of state omission is just $0.2 \%$.

Previously, the memory and time bottleneck in the performance analysis pipeline has been state space exploration. We believe that our technique shifts this bottleneck away from state space generation and onto stages later in the analysis pipeline. Future work will focus on completing the performance analysis pipeline with a parallel functional analyser and a parallel steady-state solver. The functional analyser will ensure that the generated state graph maps onto an irreducible Markov chain by eliminating transient states and by verifying that the remaining states are strongly connected. The steady-state solver will then solve the state graph's underlying Markov chain for its steady-state probability distribution using standard techniques for linear simultaneous equations.

\section{Acknowledgements}

The authors would like to thank the Imperial College Parallel Computing Centre for the use of the AP3000 distributed-memory parallel computer. The authors would also like to thank the anonymous referees for their helpful comments. William Knottenbelt gratefully acknowledges the support and funding provided by the Beit Fellowship for Scientific Research. 


\section{References}

1. M. Ajmone-Marsan, G. Conte, and G. Balbo. A class of Generalised Stochastic Petri Nets for the performance evaluation of multiprocessor systems. ACM Transactions on Computer Systems, 2:93-122, 1984.

2. S.C. Allmaier and G. Horton. Parallel shared-memory state-space exploration in stochastic modeling. Lecture Notes in Computer Science, 1253, 1997.

3. F. Basket, K.M. Chandy, R.R. Muntz, and F.G. Palacios. Open, closed and mixed networks of queues with different classes of customers. Journal of the ACM, 22:248 - 260, 1975.

4. F. Bause. Queueing Petri nets: A formalism for the combined qualitative and quantitative analysis of systems. In Proceedings of the 5th International Workshop on Petri nets and Performance Models. IEEE, October 1993.

5. P. Buchholz. Hierarchical Markovian models: Symmetries and aggregation. Performance Evaluation, 22:93-110, 1995.

6. S. Caselli, G. Conte, and P. Marenzoni. Parallel state exploration for GSPN models. In Lecture Notes in Computer Science 935: Proceedings of the 16th International Conference on the Application and Theory and Petri Nets. Springer Verlag, Turin, Italy, June 1995.

7. G. Ciardo, J. Gluckman, and D. Nicol. Distributed state-space generation of discrete-state stochastic models. INFORMS J. Comp. To appear.

8. G. Ciardo, J.K. Muppula, and K.S. Trivedi. On the solution of GSPN reward models. Performance Evaluation, 12(4):237-253, 1991.

9. G. Ciardo and K.S. Trivedi. A decomposition approach for stochastic reward net models. Performance Evaluation, 18(1):37-59, 1993.

10. E.W. Dijkstra, W.H.J. Feijen, and A.J.M. van Gasteren. Derivation of a termination detection algorithm for distributed computations. Information Processing letters, 16:217-219, June 1983.

11. A. Geist, A. Beguelin, J. Dongarra, W. Jiang, R. Manchek, and V. Sunderam. PVM Parallel Virtual Machine: A Users' Guide and Tutorial for Networked Parallel Computing. MIT Press, Cambridge, Massachussetts, 1994.

12. W. Gropp, E. Lusk, and A. Skjellum. Using MPI: Portable Parallel Programming with the Message Passing Interface. MIT Press, Cambridge, Massachussetts, 1994.

13. G.J. Holzmann. Design and Validation of Computer Protocols. Prentice-Hall, 1991.

14. G.J. Holzmann. An analysis of bitstate hashing. In Proceedings of IFIP/PSTV95: Conference on Protocol Specification, Testing and Verification. Chapman \& Hall, Warsaw, Poland, June 1995.

15. H. Ishihata, M. Takahashi, and H. Sato. Hardware of AP3000 scalar parallel server. Fujitsu Scientific and Technical Journal, 33(1):24-30, June 1997.

16. P. Kemper. Numerical analysis of superposed GSPNs. In Proc. of the Sixth International Workshop on Petri Nets and Perfromance Models, pages 52-62. IEEE Computer Society Press, 1995.

17. W.J. Knottenbelt. Generalised Markovian analysis of timed transition systems. Master's thesis, University of Cape Town, 1996.

18. U. Stern and D.L. Dill. Improved probabilistic verification by hash compaction. In IFIP WG 10.5 Advanced Research Working Conference on Correct Hardware Design and Verification Methods, 1995.

19. P. Wolper and D. Leroy. Reliable hashing without collision detection. In Lecture Notes in Computer Science 697, pages 59-70. Springer-Verlag, 1993. 